J3eA - Vol. 2 - 8 (2003).

DOI : 10.1051/bib-j3ea:2003008

\title{
Approche expérimentale d'une ligne de transmission par fibre optique
}

\author{
P. Arguel * et P. Destruel ${ }^{* *}$ (Université Toulouse III) \\ Mis en ligne le 17 juin 2003.
}

\begin{abstract}
Résumé
Cet article présente une série de trois TP permettant d'appréhender concrètement les problématiques liées au domaine des communications optiques. Chaque séance de TP est dédiée à un élément essentiel d'une ligne de transmission optique (émetteur, fibre optique, amplificateur à fibre dopée) et l'accent est mis sur l'étude d'aspects fondamentaux accessibles avec un appareillage courant et bon marché.

Mots-clés : laser à semiconducteur, fibre optique, amplificateur à fibre dopée, liaison optique, système de transmission optique, télécoms optiques.
\end{abstract}

(C) EDP Sciences, 2003.

Niveau de connaissances requis. Cet enseignement vient compléter une partie théorique représentant 16 heures d'enseignement.

Niveau des étudiants. Deuxième cycle : maîtrise EEA.

* Philippe Arguel ${ }^{1}$ est maître de conférences à l'Université Toulouse III. Il enseigne l'électricité et l'optique en DEUG, l'optoélectronique en Maîtrise de Physique et en préparation à l'Agrégation de Physique Appliquée, et les systèmes télécoms sur fibre optique en Maîtrise EEA et en IUP.

Il mène ses activités de recherche au LAAS/CNRS. Après avoir travaillé sur la conception et la technologie de diodes laser dans la filière GaAs, ses travaux s'orientent maintenant sur l'intégration photonique dans les microsystèmes.

a e-mail : arguel@laas.fr (auteur de correspondance)

** Pierre Destruel ${ }^{2}$ est professeur à l'Université Toulouse III. Il enseigne l'électricité et l'optique en DEUG, les fibres optiques pour télécoms en Maîtrise EEA, en DEA et en Formation Continue, et les isolants et l'électronique organique en DEA.

Il développe ses recherches au Laboratoire de Génie Électrique de Toulouse (LGET) où il est en charge du groupe Matériaux Organiques pour l'Optoélectronique en Génie Électrique. Il étudie plus particulièrement les Diodes Electroluminescentes Organiques et les Cellules Photovoltaïques Organiques.

email : pierre.destruel@lget.ups-tlse.fr

\footnotetext{
${ }^{1}$ LAAS/CNRS, 7 avenue du Colonel Roche, F-31077 Toulouse Cedex 4, France.

${ }^{2}$ LGET/Université Toulouse III, Laboratoire de Génie Électrique, 118 route de Narbonne, F-31062 Toulouse Cedex, France.
} 


\section{Introduction}

Au cours de la dernière décennie, les télécommunications optiques ont connu un développement sans précédent. Dans ce contexte, nous avons mis en place, à l’Université Paul Sabatier (Toulouse III), une série d'enseignements pratiques permettant aux étudiants de la filière EEA d'appréhender concrètement les problématiques liées à ce domaine. Les moyens relativement modestes mis en œuvre nous ont conduits, dans un premier temps, à privilégier l'étude d'aspects fondamentaux accessibles avec un appareillage courant et bon marché.

L'étude expérimentale que nous proposons se décompose en trois séances de travaux pratiques $(3 \times 3$ heures $)$ dont chacune est relative à l'étude d'un élément fondamental d'une ligne de transmission optique : l'émetteur, la fibre optique, et l'amplificateur optique à fibre dopée.

Le récepteur, quant à lui, fait partie intégrante de tous les bancs de manipulation et n'est pas étudié de façon spécifique pour le moment.

\section{2. Étude d'un émetteur}

L'objet de cette manipulation est de déterminer les caractéristiques essentielles d'un laser à semiconducteur et d'établir ses conditions optimales de fonctionnement pour l'application visée. Dans un but à la fois pédagogique, de sécurité et d'économie, nous avons écarté les émetteurs infrarouges à grande pureté spectrale (émettant typiquement à 1,3 et 1,55 microns pour les télécoms optiques) et avons choisi de travailler avec des diodes laser conventionnelles émettant à la longueur d'onde de 660 nm (Fig. 1 à Fig. 4).

\subsection{Présentation du composant étudié}
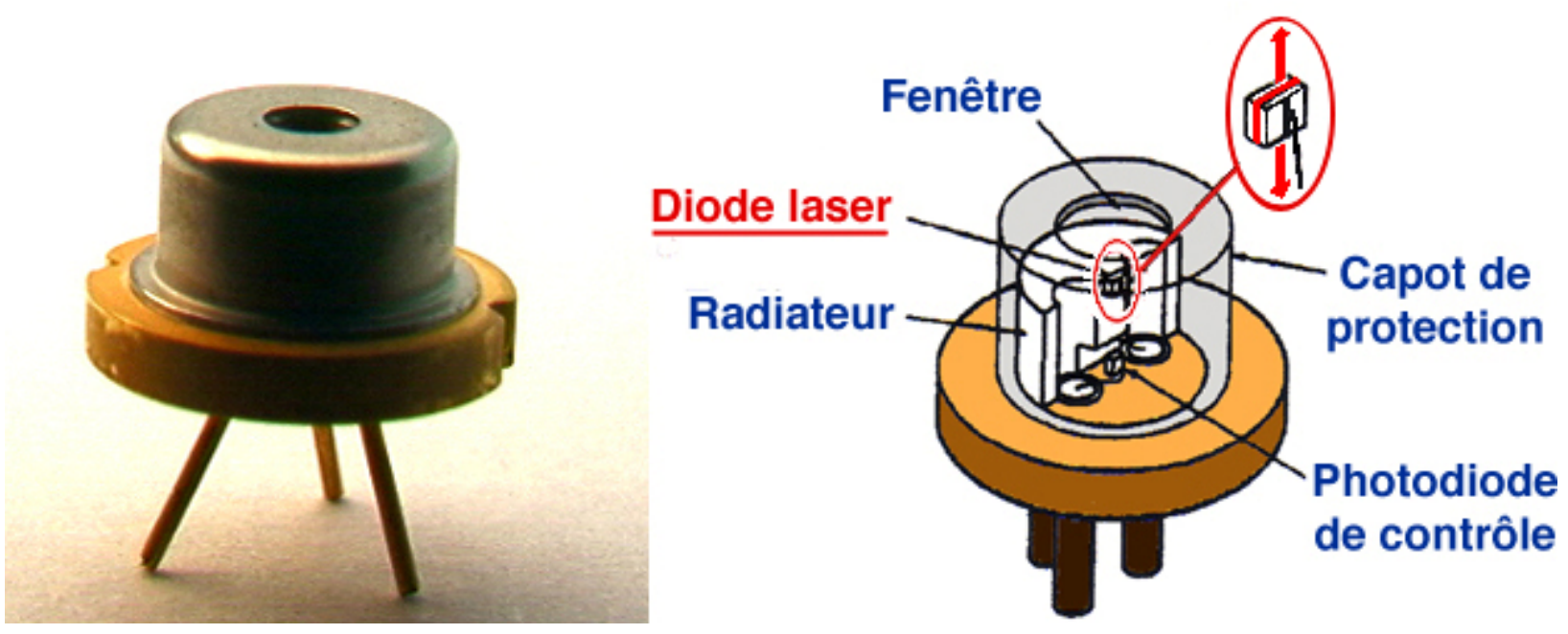

Fig. 1. Vue générale d'une tête laser (gauche) et agencement de ses différents éléments (droite). 


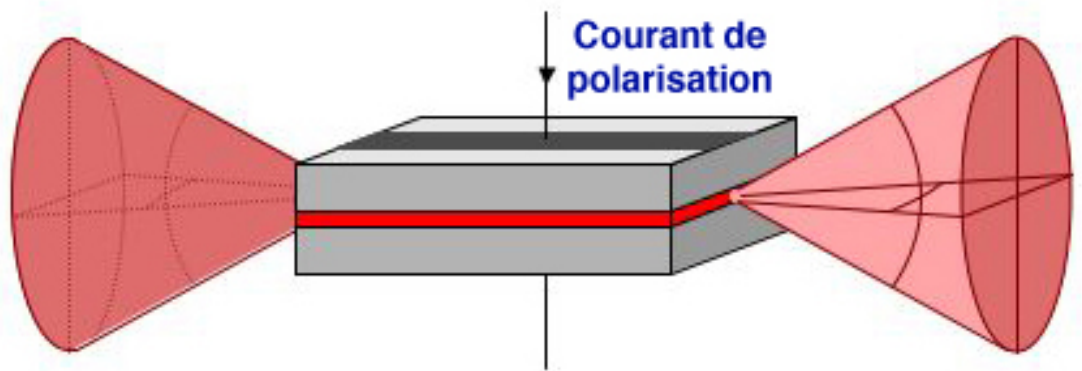

Fig. 2. Représentation schématique du composant laser à semiconducteur et des deux faisceaux lumineux émis sur les faces avant et arrière. La région active du composant est indiquée en rouge.

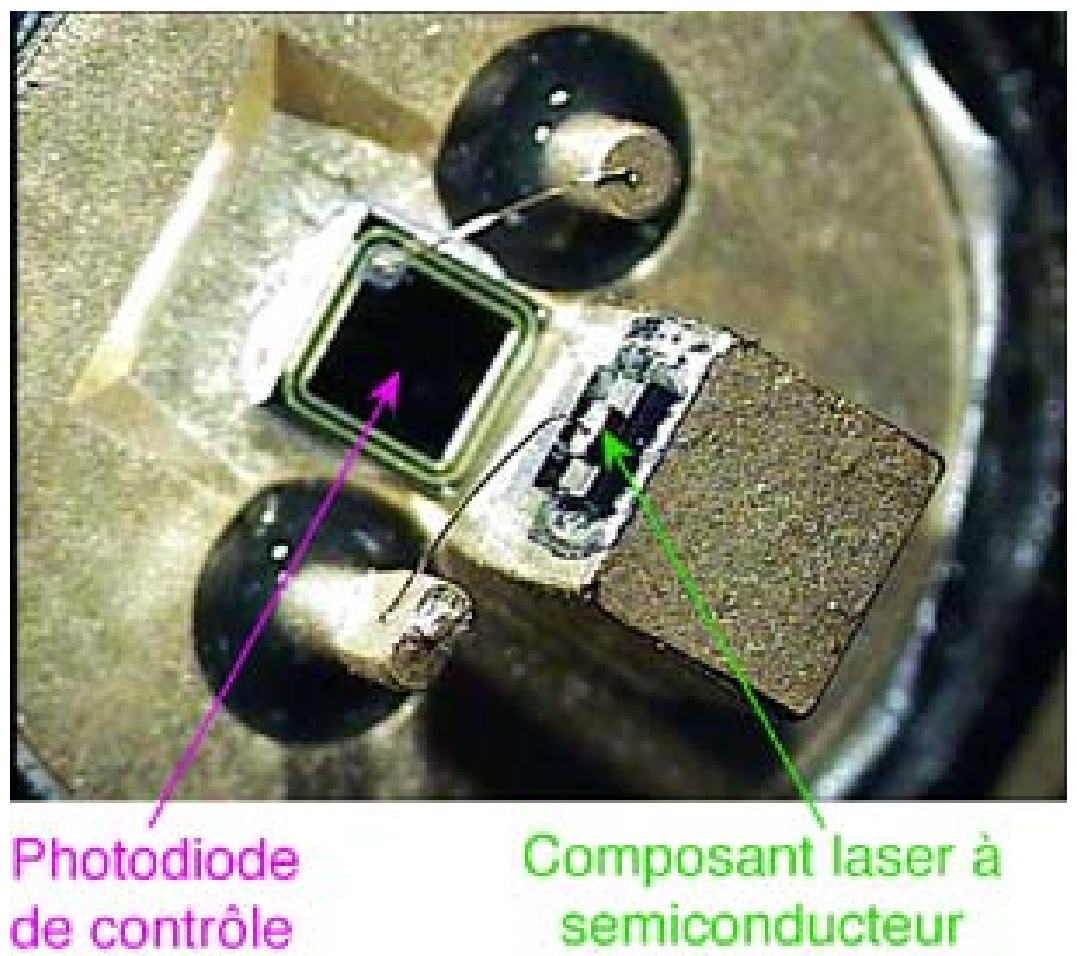

Fig. 3. Vue détaillée des éléments constituant la tête laser (placés sous le capot de protection).

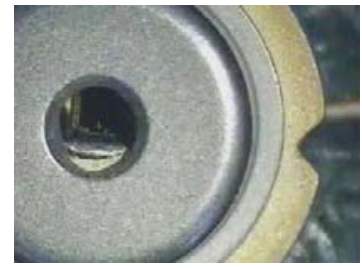

Fig. 4. Observation de la diode laser au travers de la fenêtre de sortie.

Remarque : Le terme «Diode laser » est utilisé indifféremment pour désigner le composant laser à semiconducteur ou la tête laser, dans son ensemble, renfermant également la photodiode de contrôle.

L'approche expérimentale proposée se décompose en trois étapes correspondant aux principales caractéristiques de ce type de composants et elle se termine par une étude générale des propriétés de modulation des diodes laser. 
Le matériel mis en œuvre pour cette manipulation est composé de deux diodes laser, deux alimentations en courant, un module à effet Peltier avec son alimentation, deux photodiodes amplifiées, un goniomètre, un générateur de signaux, un oscilloscope et deux voltmètres.

- $\quad$ les propriétés optiques de la diode laser ;

- $\quad$ ses caractéristiques $\mathrm{V}(\mathrm{I})$ et $\mathrm{P}(\mathrm{I})$;

- $\quad$ son comportement en température ;

- $\quad$ son comportement en modulation.

\subsection{Propriétés optiques}

La diode laser est étudiée ici à température ambiante. Elle est montée au centre d'un barillet dont le plan est vertical et elle peut ainsi tourner sur elle-même en émettant toujours son rayonnement selon un axe horizontal. De plus, elle est située sur l'axe de rotation du bras du goniomètre qui porte une photodiode amplifiée (Fig. 5).

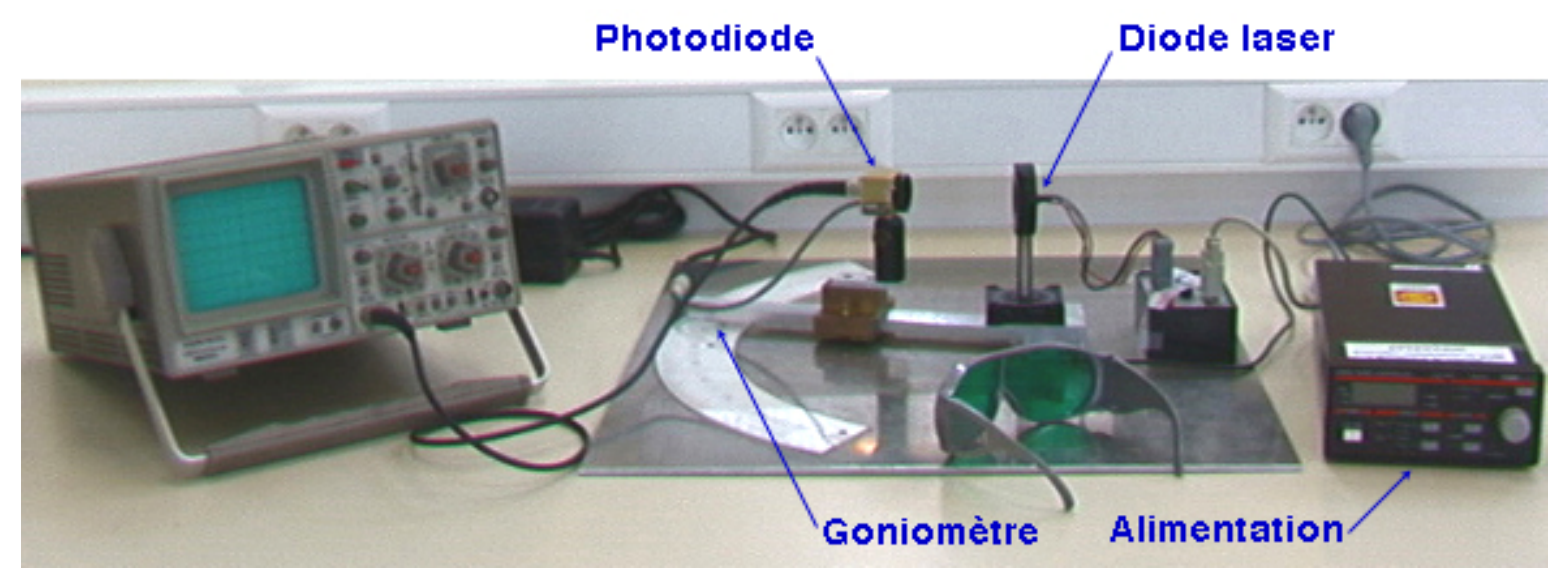

Fig. 5. Vue d'ensemble du montage goniométrique.

L'étude débute par une observation du faisceau à l'aide d'un écran, les caractéristiques de la diode utilisée permettant une observation dans d'excellentes conditions de sécurité. La transition entre le régime électroluminescent et le régime laser apparaît alors de façon évidente lorsque le courant de polarisation augmente. L'existence d'un courant de seuil est ainsi « visualisée » et son ordre de grandeur est noté. On observe également que la trace du faisceau sur l'écran est fortement elliptique (Fig. 6), on en détermine les raisons et on en déduit l'orientation du composant sur son support. Pour la suite, cette orientation est facilement ajustable à l'aide du barillet.
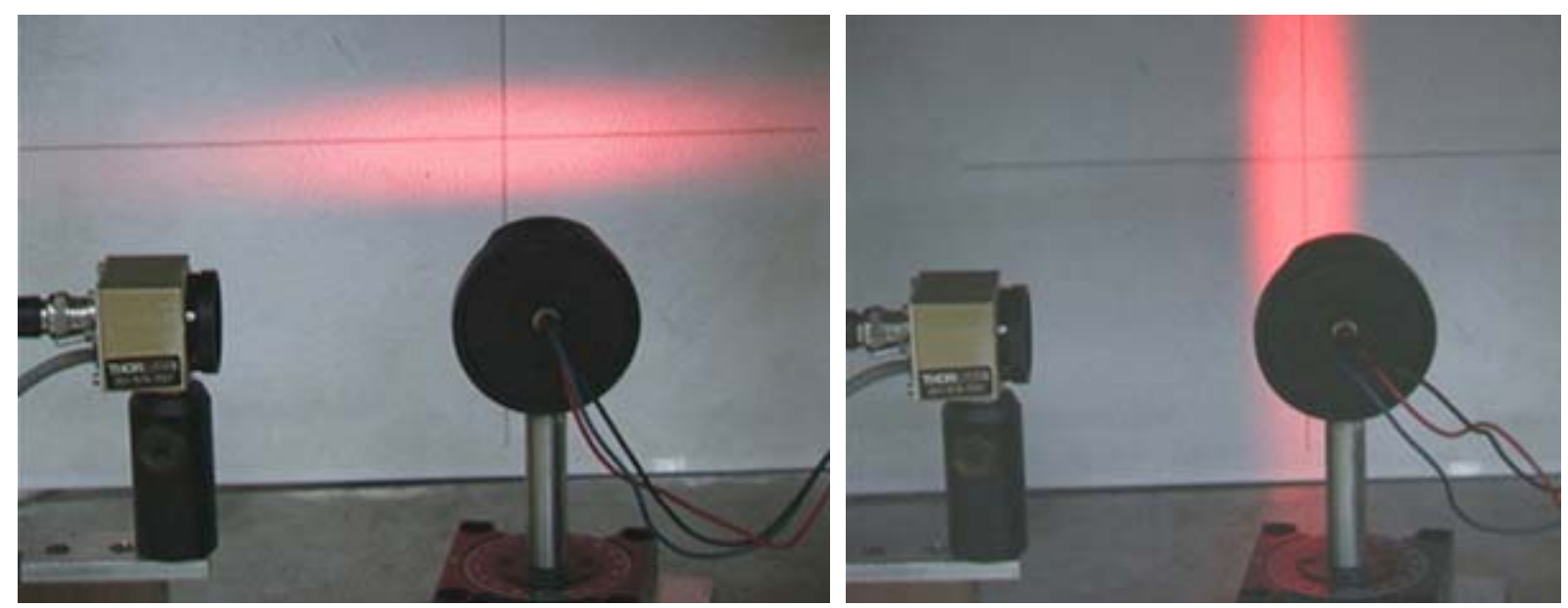

Fig. 6. Observation de la trace du faisceau laser sur l'écran. Le plan de la région active de la diode laser peut être orienté verticalement (gauche) ou horizontalement (droite) à l'aide du barillet. 


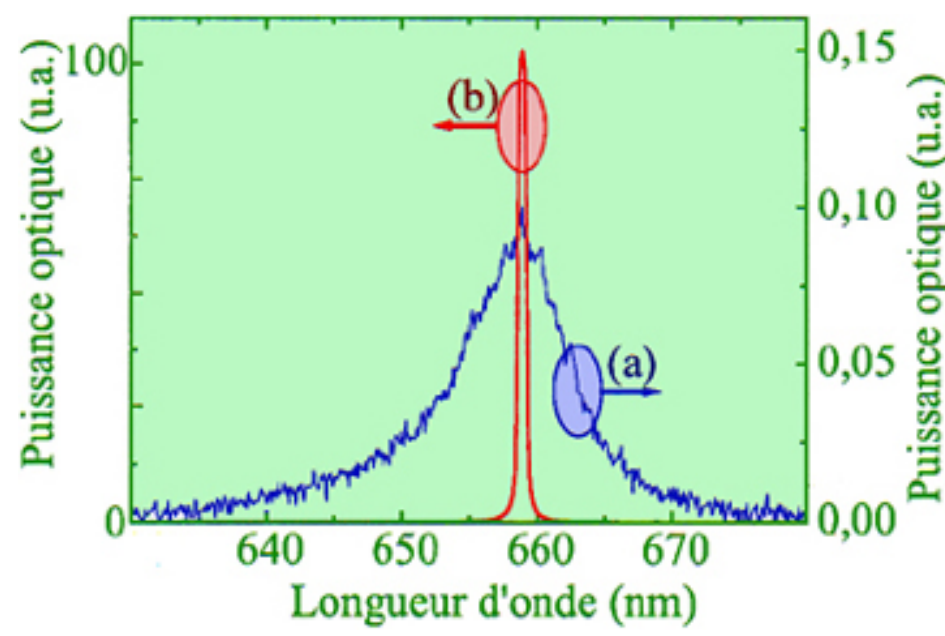

Le relevé du spectre d'émission de la diode laser n'est pas réalisé actuellement par les étudiants car cela demanderait un matériel coûteux dont le banc de manipulation n'est pas équipé. Toutefois, nous avons effectué préalablement ce relevé en laboratoire et nous le fournissons aux étudiants pour discussion (Fig. 7).

Fig. 7. Spectre d'émission pour le régime électroluminescent (a) et le régime laser (b).

À l'aide de la photodiode portée par le goniomètre, on détermine le diagramme de rayonnement de la diode laser selon le grand axe et le petit axe de l'ellipse observée précédemment (Fig. 8). Ces deux axes correspondent respectivement à la direction perpendiculaire et parallèle au plan de la région active du composant. La mesure de la largeur à mi-hauteur du diagramme de rayonnement selon ces deux directions permet alors une estimation des dimensions transverses et latérales du guide optique entourant la région active.

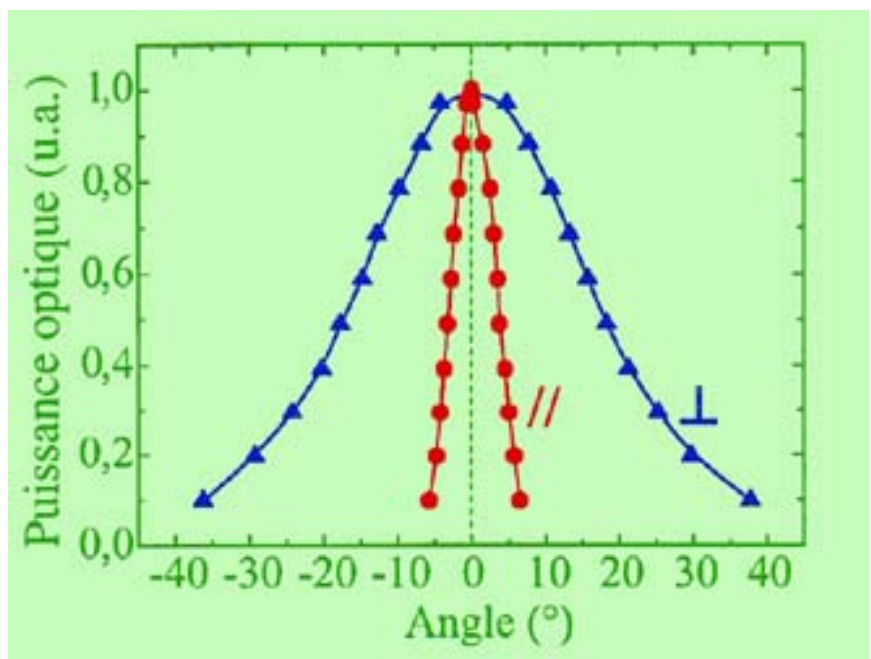

Fig. 8. Diagramme de rayonnement selon la direction perpendiculaire (en bleu) et parallèle (en rouge) au plan de la région active.

\subsection{Caractéristiques $V(I)$ et $P(I)$}

Cette étape a pour objet le relevé et l'analyse, à température donnée, de deux caractéristiques essentielles des diodes laser : la caractéristique « tension/courant», dite également V(I), et la caractéristique « puissance optique/courant », dite $\mathrm{P}(\mathrm{I})$. Ces mesures sont effectuées à l'aide du dispositif expérimental présenté ci-dessous (Fig. 9). 


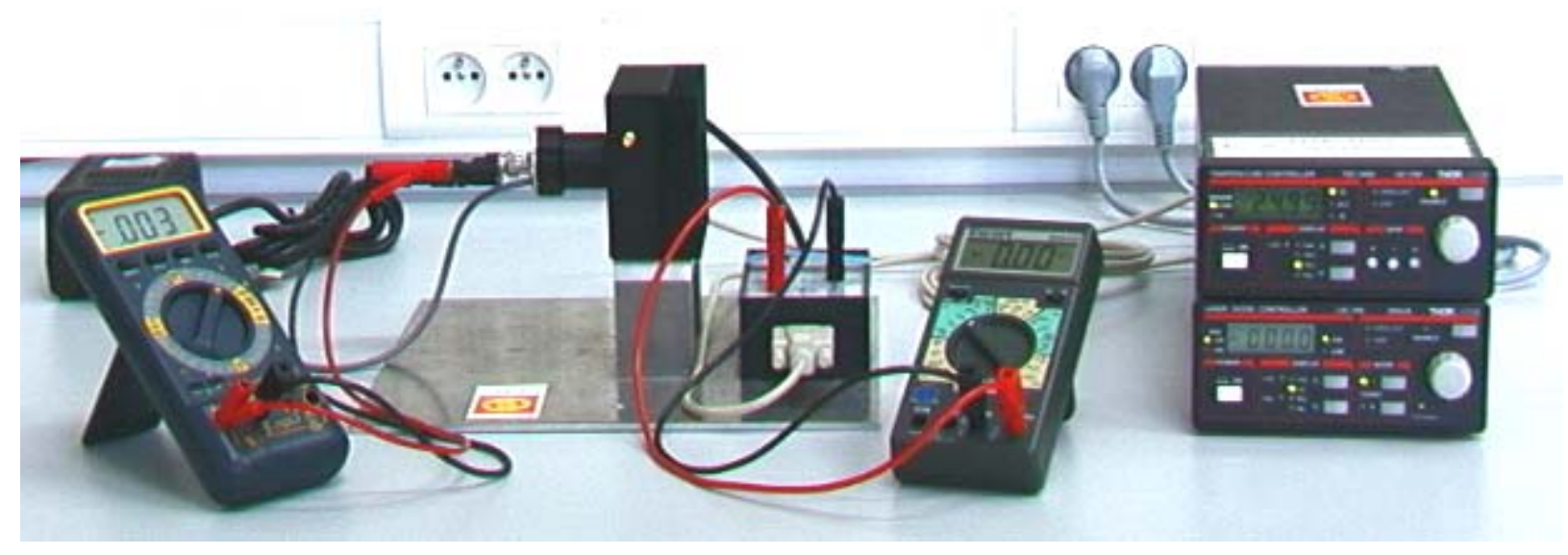

Fig. 9. Montage expérimental permettant le relevé des caractéristiques $\mathrm{P}(\mathrm{I})$ et V(I). Au centre : module à effet Peltier contenant la diode laser à étudier.

Le relevé expérimental de $\mathrm{V}(\mathrm{I})$ est obtenu grâce à un boîtier de contrôle (Fig. 10) que nous avons inséré dans le circuit d'alimentation de la diode laser. Ce relevé met en évidence la présence d'une tension de coude $\mathrm{V}_{\mathrm{c}}$ et conduit à la détermination de la résistance série $\mathrm{R}_{\mathrm{s}} \mathrm{du}$ composant (Fig. 11). La caractéristique électrique ainsi obtenue peut alors être comparée à celle d'une diode « électronique » ordinaire bien connue des étudiants.

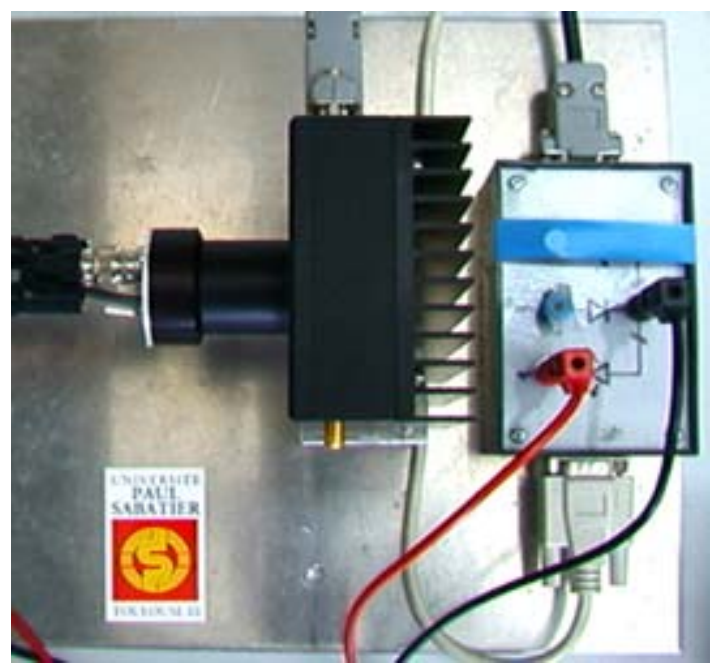

Fig. 10. Vue détaillée du boîtier de contrôle permettant la mesure de la chute de potentiel aux bornes de la diode laser.

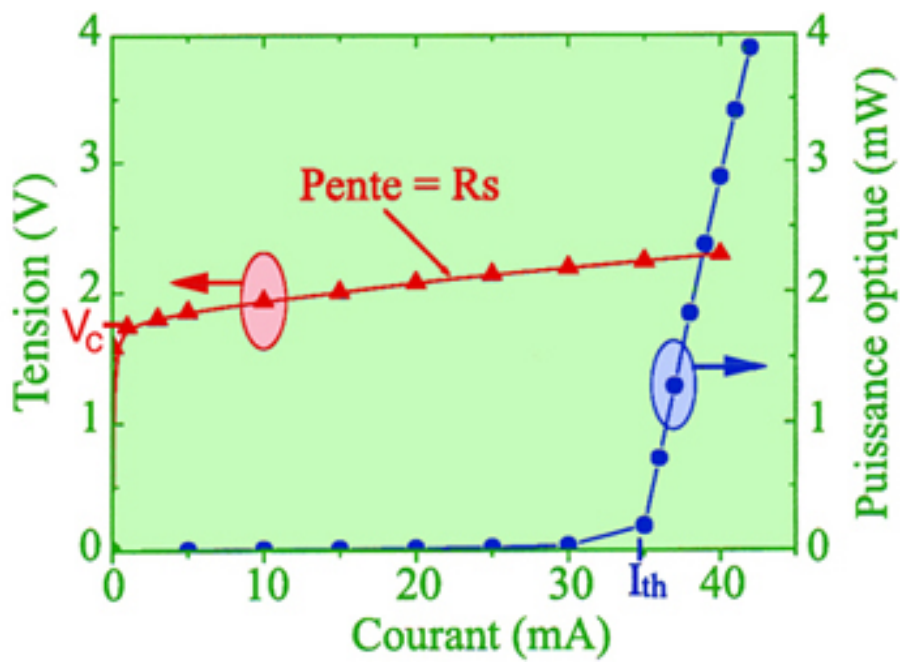

Fig. 11. Relevé expérimental des caractéristiques V(I) et P(I).

Le relevé de $\mathrm{P}(\mathrm{I})$, quant à lui, est obtenu au moyen d'une simple photodiode préalablement étalonnée en puissance par l'équipe pédagogique. Après avoir noté le caractère linéaire du régime laser, on détermine avec précision la valeur du courant de seuil $\mathrm{I}_{\text {th }}$ de la diode laser pour la température considérée (Fig. 11). Enfin, les rendements quantiques différentiel et externe sont déterminés pour deux points de fonctionnement distincts et leurs valeurs relatives sont commentées.

\subsection{Comportement en température}

Dans cette partie, on met en évidence l'influence de la température de fonctionnement sur les performances de la diode laser à l'aide du montage présenté dans le paragraphe précédent (Fig. 9). Pour cela, on relève les valeurs du courant de seuil correspondant à différentes températures. On note alors une évolution de ce courant qui est régie par l'expression empirique :

$$
\mathrm{I}_{\mathrm{th}}\left(\mathrm{T}_{2}\right)=\mathrm{I}_{\mathrm{th}}\left(\mathrm{T}_{1}\right) \exp \left[\left(\mathrm{T}_{2}-\mathrm{T}_{1}\right) / \mathrm{T}_{0}\right]
$$


où $T_{2}$ et $T_{1}$ sont deux températures de fonctionnement et $T_{0}$ est le coefficient de température (exprimé en kelvin) caractérisant la diode laser étudiée. Le tracé du logarithme népérien du courant de seuil en fonction de la température de fonctionnement conduit alors naturellement à la détermination graphique du coefficient $\mathrm{T}_{0}$ (Fig. 12).

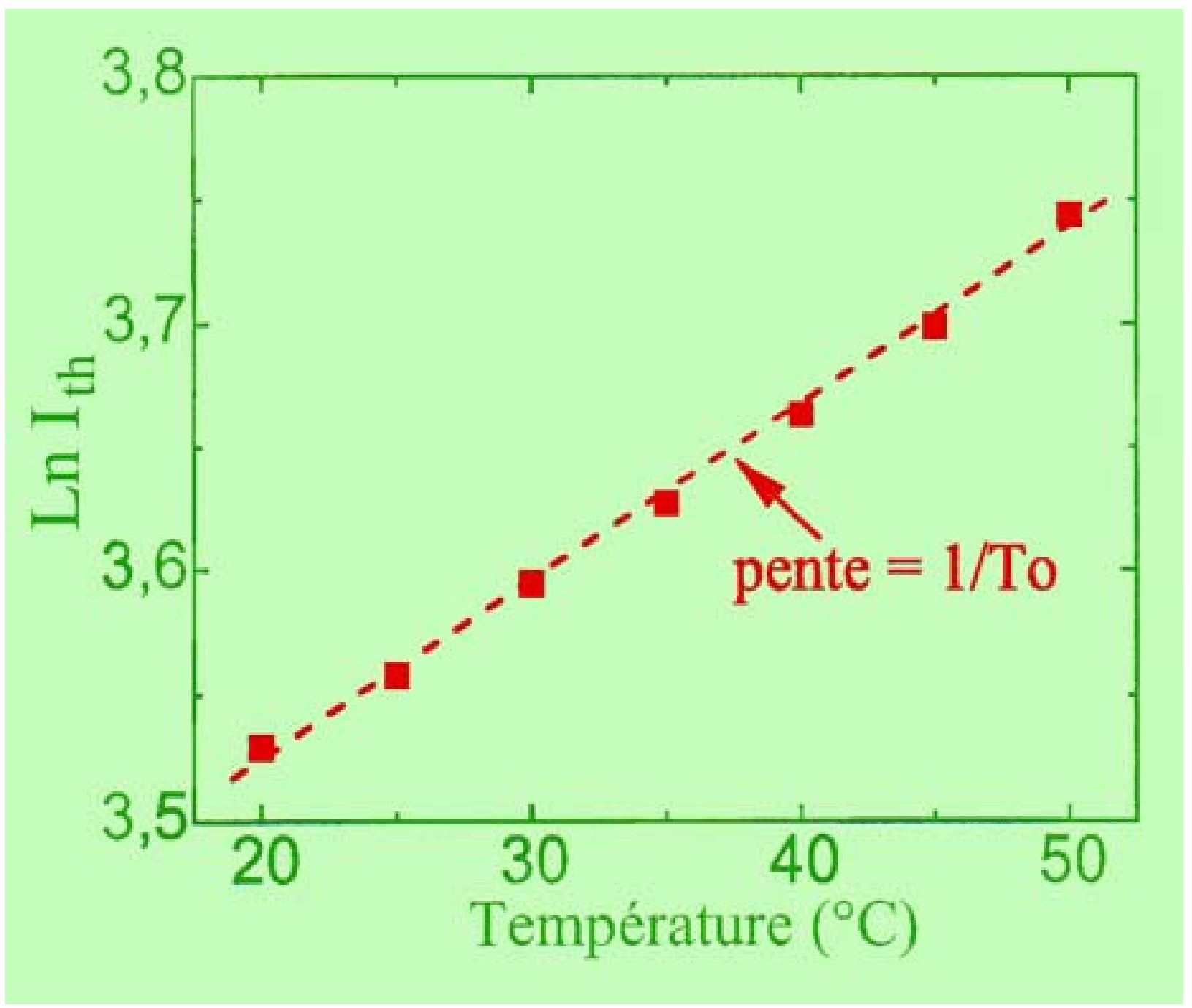

Fig. 12. Variation du courant de seuil en fonction de la température de fonctionnement.

Cette partie de l'étude se termine par une comparaison de différentes valeurs de $\mathrm{T}_{0}$ données dans la littérature et, enfin, on souligne l'influence de la température de fonctionnement sur la longueur d'onde d'émission. 


\subsection{Comportement en modulation}

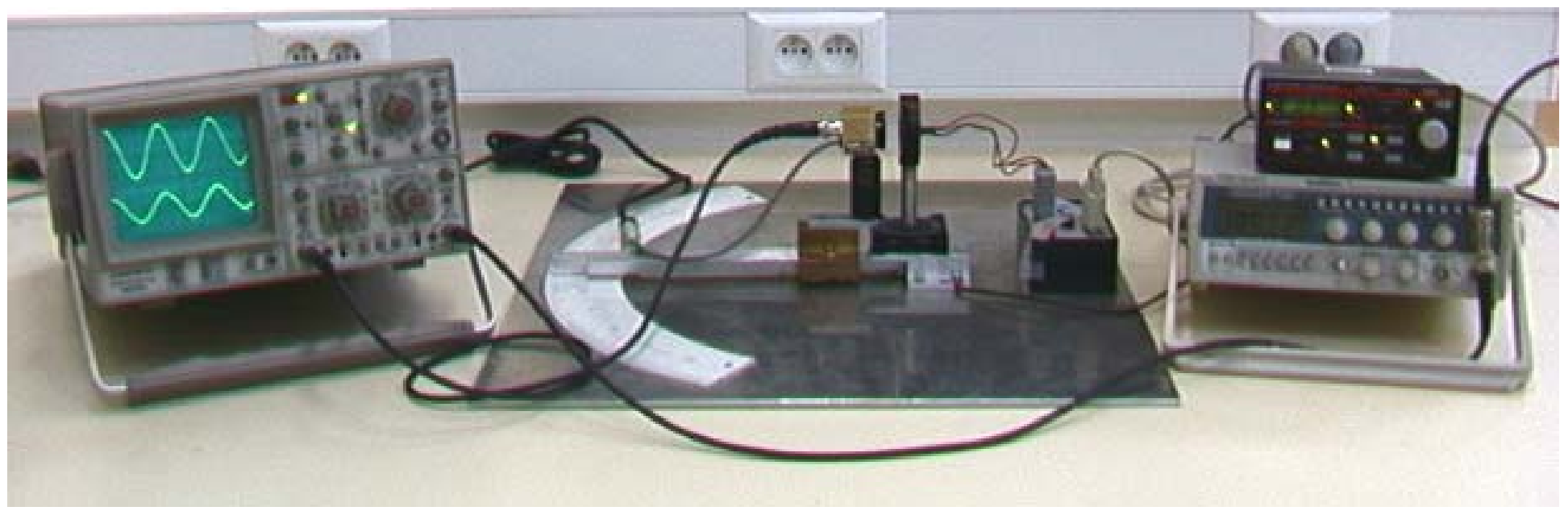

Fig. 13. Montage expérimental permettant l'étude du comportement de la diode laser en modulation.

La modulation de la puissance optique délivrée par la diode laser est directement obtenue par l'utilisation d'un générateur de fonctions connecté à l'entrée « modulation » de l'alimentation (Fig. 13). À l'aide de l'oscilloscope (Fig. 14), on observe le comportement du système pour différentes valeurs du courant de polarisation et pour des signaux de modulation variés (formes, amplitudes, fréquences). On détermine ensuite les conditions nécessaires pour une modulation numérique et pour une modulation analogique performantes.
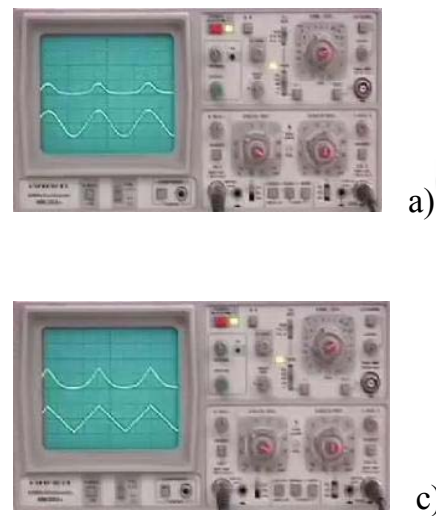

c)

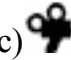

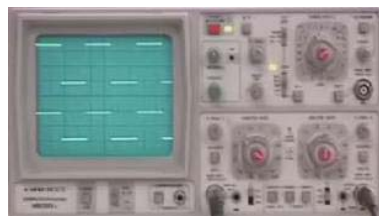
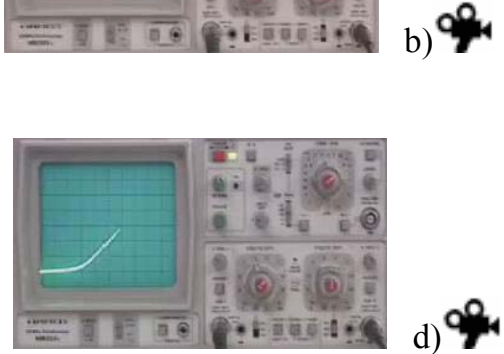

d)

Fig. 14. Observations à l'oscilloscope du comportement de la diode laser en modulation.

Sur les vidéos a), b) et c) de la figure 14, on visualise le signal délivré par le générateur de fonctions sur la partie inférieure de l'écran et la réponse de la diode laser sur la partie supérieure. Cette réponse varie avec la valeur de la composante continue du courant de polarisation qui varie elle-même lentement à proximité du courant de seuil. On peut observer l'existence d'une plage de linéarité dans la réponse, exploitable dans le cadre d'une modulation analogique.

La vidéo d) de la figure 14 représente en mode XY la variation de la puissance optique (Y) lorsqu'on applique une modulation triangulaire sur le courant de polarisation $(\mathrm{X})$ : on retrouve ici la caractéristique $\mathrm{P}(\mathrm{I})$ étudiée précédemment. 


\section{3. Étude de fibres optiques}

Pour les raisons déjà évoquées, cette étude se fait également au moyen de diodes laser émettant une longueur d'onde de $660 \mathrm{~nm}$.

Le but de la manipulation est de déterminer les principales caractéristiques de différentes fibres optiques et de liaisons simples. La démarche expérimentale que nous proposons ici permet d'aborder successivement les notions d'ouverture numérique, d'atténuations (à l'injection, de connexion, linéique), de bilan énergétique et de propagation d'impulsions le long d'une fibre optique multimode. On conclut l'étude par une manipulation relative aux fibres monomodes.

Le matériel utilisé pour ces travaux se compose d'une diode laser montée dans un système de couplage réglable, une alimentation en courant, différentes fibres courtes multimodes (jarretières d'un mètre), un rouleau de 500 mètres de fibre multimode connectorisée, une fibre monomode (jarretière de 5 mètres), un puissance-mètre, un goniomètre portant une photodiode amplifiée, un générateur de signaux et un oscilloscope.

Nous étudierons successivement :

- l'ouverture numérique ;

- l'atténuation;

- la propagation ;

- le cas d'une fibre monomode.

\subsection{Ouverture numérique}

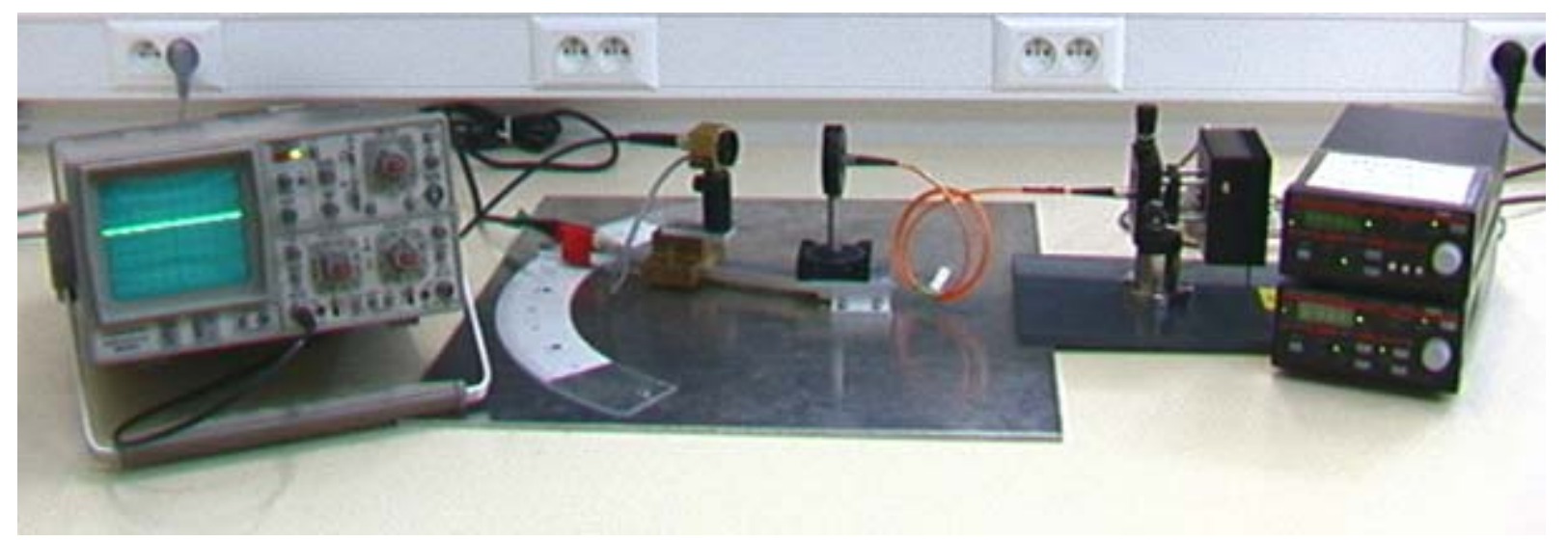

Fig. 15. Montage goniométrique présenté avec une jarretière d'un mètre (propagation hors équilibre modal).

Dans la démarche expérimentale permettant de déterminer l'ouverture numérique d'une fibre optique multimode, les étudiants sont confrontés immédiatement à la notion d'équilibre modal. En effet, le premier montage que nous proposons consiste à injecter le faisceau émis par la diode laser dans une fibre courte (jarretière d'un mètre) dont l'autre extrémité est connectée au support placé sur l'axe de rotation du bras du goniomètre (Fig. 15).

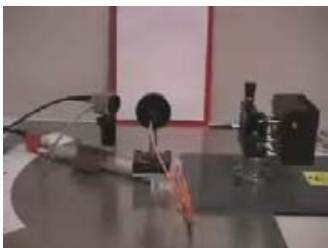

Fig. 16. Observation du changement de répartition modale en fonction du trajet de la fibre et des conditions d'injection.
À ce stade, une observation directe du faisceau émergent à l'aide d'un simple écran indique clairement que la répartition de puissance optique dans la « tache » lumineuse est très bruitée. De plus, cette répartition est fonction du trajet de la fibre elle-même et des conditions d'injection du rayonnement dans celle-ci (Fig. 16). 
L'étape suivante consiste donc à proposer un montage permettant d'atteindre la situation d'équilibre modal, condition nécessaire pour la détermination de l'ouverture numérique de la fibre (Fig. 17).

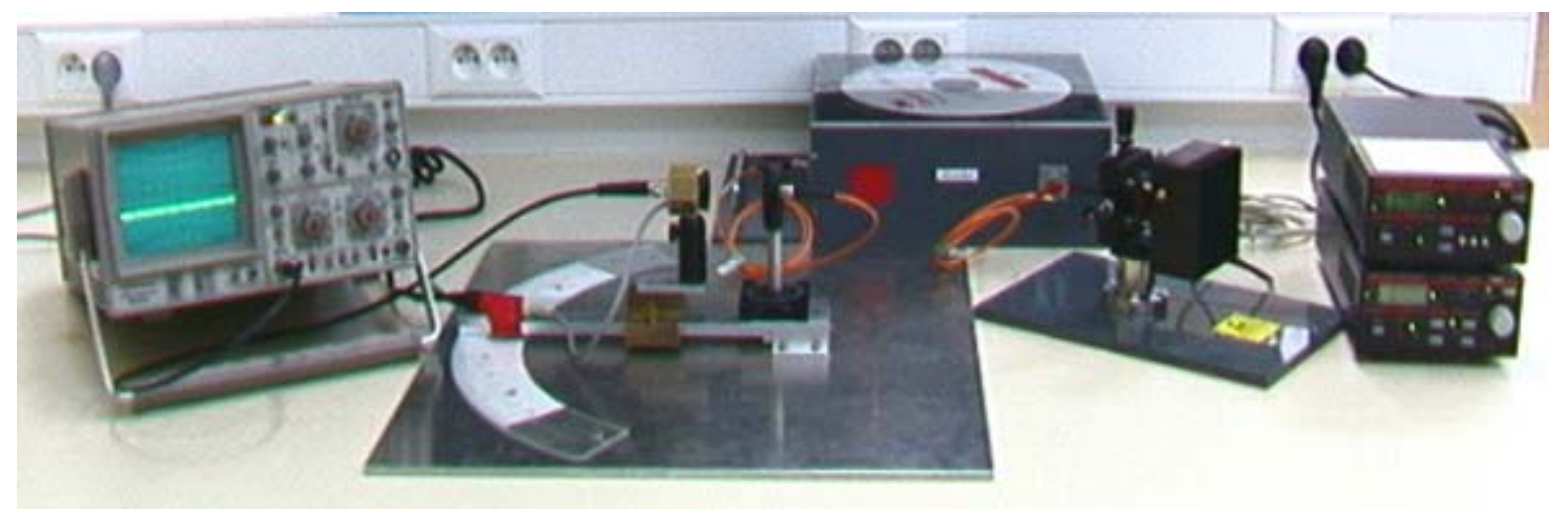

Fig. 17. Montage goniométrique permettant la mesure de l'ouverture numérique de la fibre (propagation à l'équilibre modal).

L'utilisation du rouleau de 500 mètres de fibre en amont de la fibre considérée précédemment permet alors d'effectuer la mesure de l'ouverture numérique dans de bonnes conditions, les deux fibres étant identiques. L'opération est ensuite renouvelée avec une deuxième jarretière dont l'ouverture numérique a une valeur inférieure à celle de la jarretière précédente. À l'issue de cette nouvelle mesure, une justification a posteriori de la démarche utilisée est alors demandée aux étudiants.

Pour faciliter les comparaisons et les interprétations ultérieures, les deux jarretières optiques que nous avons choisies ici présentent des ouvertures numériques respectivement égales à 0,39 et à 0,25 .

\subsection{Atténuations}

L'objet de cette partie est de faire découvrir aux étudiants les différents facteurs provoquant un affaiblissement de la puissance optique le long d'une liaison par fibre. Ici, on utilise systématiquement le « $\mathrm{dBm} »$ dans tous les calculs en précisant sa définition et en mettant en garde sur de possibles confusions avec des grandeurs électriques.

La première manipulation est relative aux pertes à l'injection. Elle consiste à injecter le rayonnement de la diode laser dans une jarretière et à mesurer la puissance optique en sortie de celle-ci. Les pertes de couplage entre la fibre et le récepteur étant connues et l'atténuation due à la propagation le long d'un mètre de fibre étant négligée, on en déduit les pertes totales dues au système d'injection. On peut alors dissocier les deux composantes de ces pertes : les pertes dues aux lentilles et diaphragmes internes au système de couplage, et les pertes d'injection localisées sur la face d'entrée de la fibre. Une mesure de ces dernières pertes pour les deux jarretières utilisées dans le paragraphe précédent met en évidence l'influence de l'ouverture numérique d'une fibre sur les pertes à l'injection (Fig. 18). 


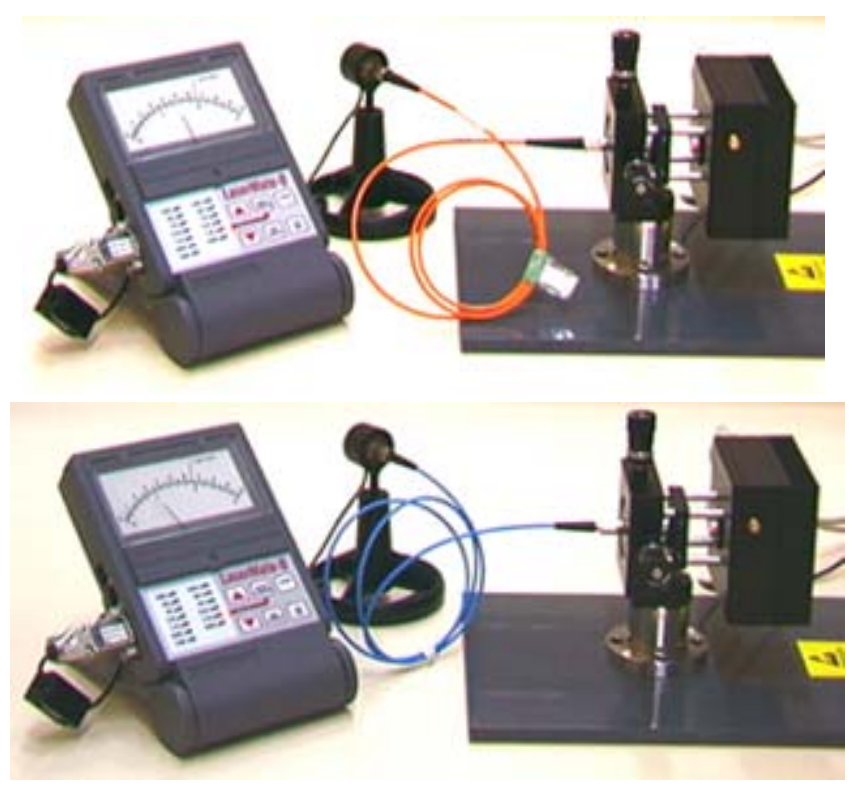

Fig. 18. Mesure des pertes à l'injection pour des fibres d'ouvertures numériques différentes (gauche : $\mathrm{ON}=0,39$; droite : $\mathrm{ON}=0,25$ ).

La manipulation suivante concerne les pertes aux connecteurs entre deux fibres multimodes (Fig. 19). Les deux jarretières sont reliées entre elles et les pertes provoquées par la traversée du connecteur sont mesurées pour les deux sens de propagation. On mesure d'abord ces pertes en injectant la lumière directement dans les jarretières (hors équilibre modal) et on reprend les mesures après avoir inséré le rouleau de 500 mètres de fibre en amont des jarretières (en équilibre modal).

Une discussion des résultats ainsi obtenus est alors demandée.

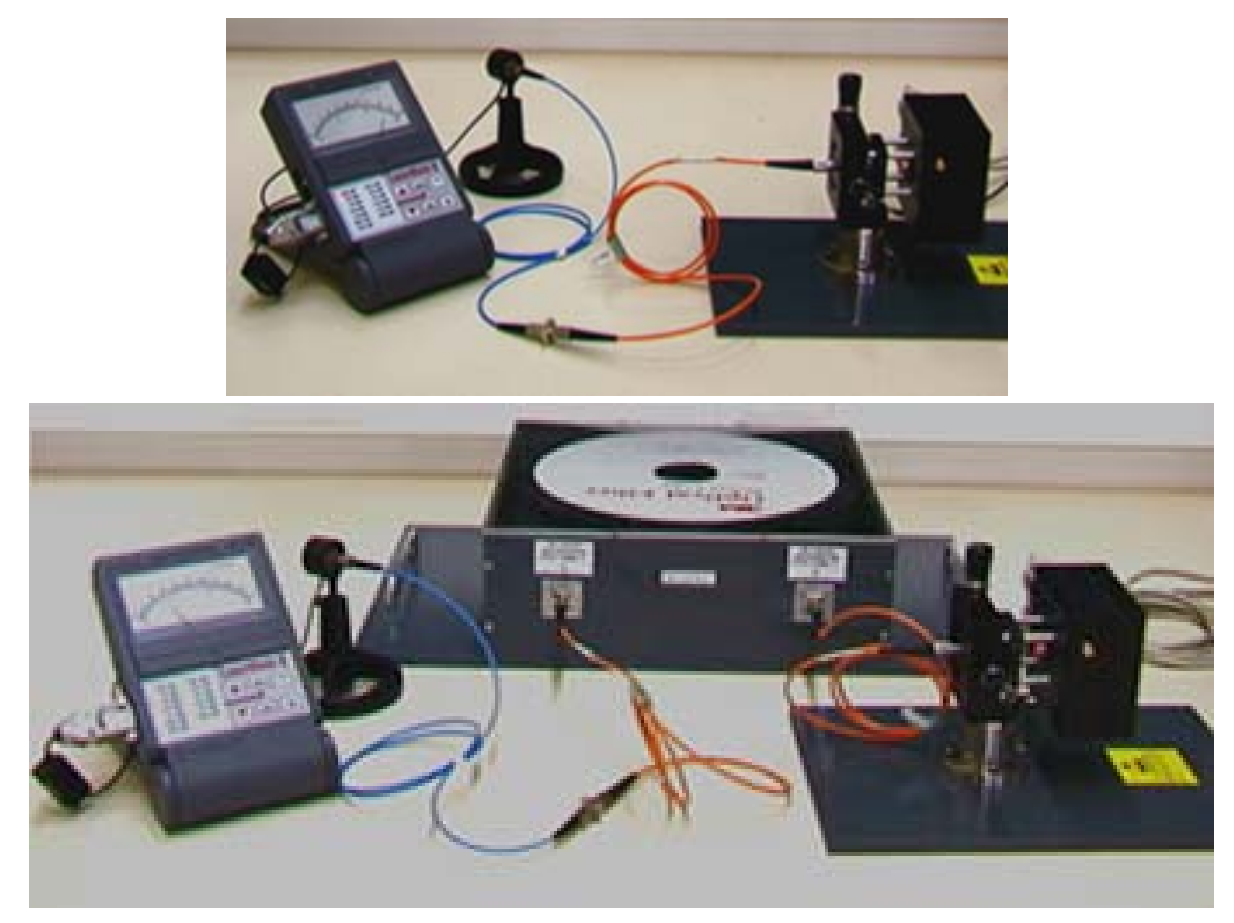

Fig. 19. Mesure des pertes de connection (gauche : hors équilibre modal ; droite : en équilibre modal).

Après avoir mesuré les pertes à l'injection et les pertes aux connecteurs, l'étape suivante concerne la détermination du coefficient d'atténuation linéique de la fibre optique constituant le rouleau. La valeur de ce 
coefficient est déduite aisément de l'expression du bilan énergétique de la liaison qui se réduit maintenant à une équation du premier degré à une inconnue. La valeur obtenue (environ $13 \mathrm{~dB} / \mathrm{km}$ ) est comparée aux valeurs de la littérature et les raisons de l'écart sont analysées.

\subsection{Propagation d'impulsions lumineuses}

On utilise ici le rouleau de 500 mètres de fibre optique et on connecte le générateur de fonctions à l'alimentation de la diode laser et à l'oscilloscope. La sortie de la fibre est reliée à la photodiode amplifiée qui est connectée également à l'oscilloscope.

La première étape consiste à visualiser le passage du régime électroluminescent au régime laser et à estimer la valeur du courant de seuil de la diode laser utilisée.

Ensuite, on superpose un signal carré au courant de polarisation et on compare les signaux délivrés par le générateur de fonctions et ceux reçus par la photodiode placée en sortie de fibre. Le retard du signal de sortie de fibre par rapport au signal du générateur est alors composé d'un retard « optique », dû au temps de propagation dans le rouleau de fibre, et d'un retard « électronique », dû essentiellement à la qualité de l'alimentation utilisée (Fig. 20).
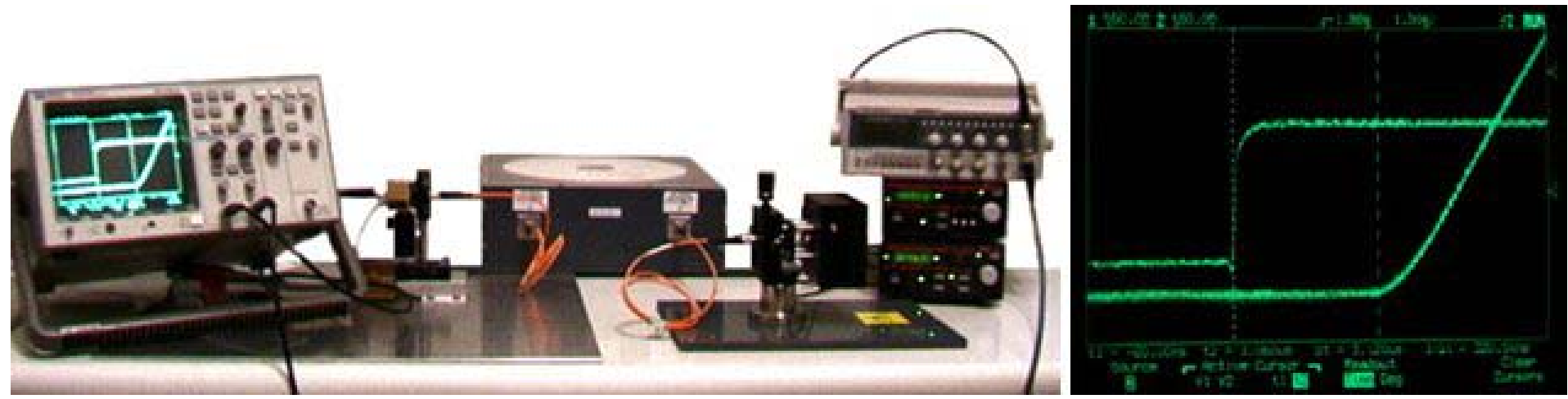

Fig. 20. Mesure du retard total « optique + électronique ».
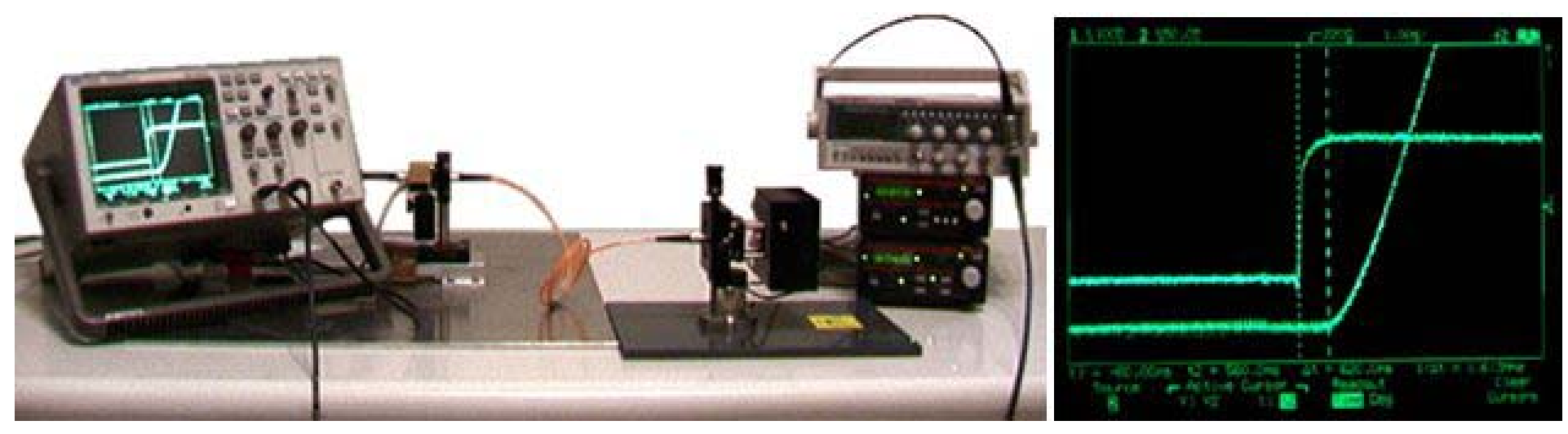

Fig. 21. Mesure du retard « électronique ».

Après avoir mesuré ce retard « électronique » à l'aide d'une jarretière d'un mètre (Fig. 21), on en déduit le temps de propagation des impulsions le long des 500 mètres du rouleau de fibre.

À ce stade, on peut se poser la question du calcul de l'indice de réfraction du cœur de la fibre optique multimode. Il est donc demandé aux étudiants de procéder à cette détermination en l'accompagnant d'un calcul d'incertitude permettant de juger de sa validité. Le résultat montre que la valeur de l'indice est déterminée ici avec une 
incertitude absolue supérieure à l'écart caractéristique entre les indices de cœur et de gaine de fibres optiques du même type.

\subsection{Fibre optique monomode}

La fibre optique utilisée dans cette dernière partie présente une longueur d'onde de coupure de 620 nm et une ouverture numérique égale à 0,12 .

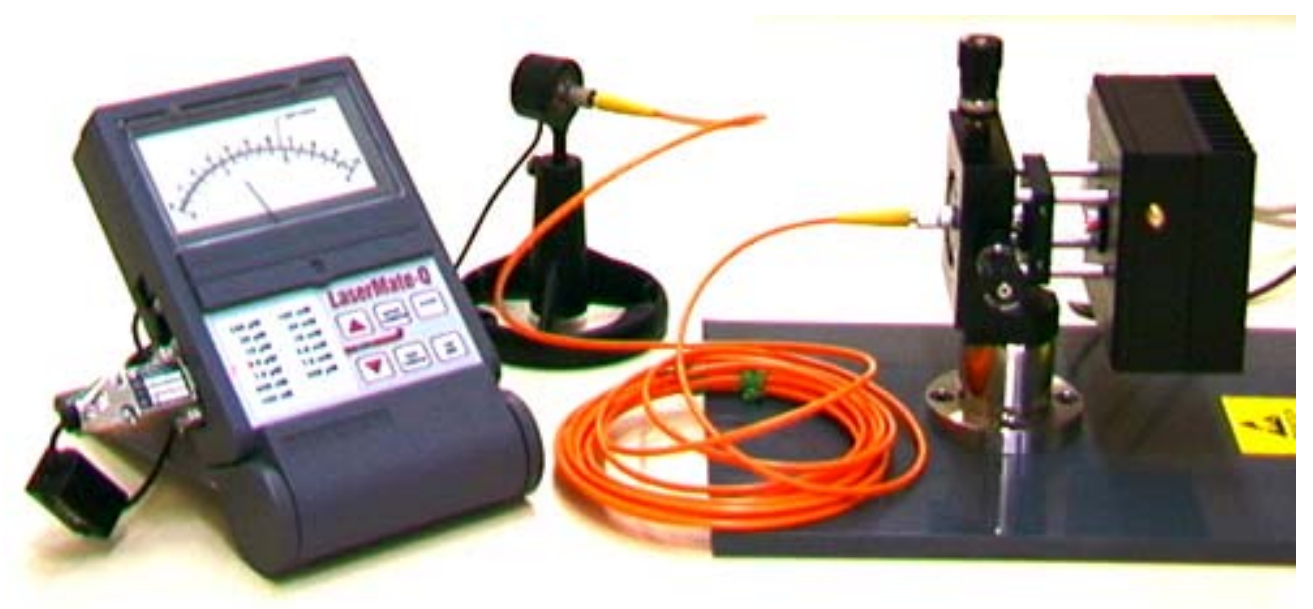

Fig. 22. Mesure des pertes d'injection dans une fibre monomode.

La manipulation proposée consiste uniquement à estimer les pertes d'injection dans cette fibre (Fig. 22). On rencontre alors de grandes difficultés pour obtenir un couplage au moyen du matériel dont on dispose et on relève des valeurs de pertes à l'injection très importantes. Cette manipulation de base permet ainsi d'appréhender les contraintes liées à l'utilisation de fibres monomodes, et elle permet de mieux apprécier les performances présentées par les systèmes de télécommunications optiques.

On termine cette approche par le calcul du diamètre du cœur de la fibre monomode que l'on compare à celui des fibres multimodes utilisées précédemment.

\section{4. Étude d'un amplificateur optique à fibre dopée à l'erbium}

Contrairement aux deux études précédentes pour lesquelles nous utilisions des diodes laser émettant un rayonnement à la longueur d'onde de $660 \mathrm{~nm}$, nous allons présenter ici des travaux menés à l'aide d'un matériel spécifique aux télécoms optiques: l'amplificateur à fibre dopée à l'erbium. Il s'agit d'un équipement commercialisé par la société IDIL, comprenant divers éléments pouvant être reliés entre eux par des fibres monomodes selon des configurations variables en fonction des manipulations envisagées (amplificateur à fibre, laser à fibre, source superfluorescente...).

Dans le cadre de cette étude, nous nous intéresserons uniquement aux éléments nécessaires à la réalisation et à la caractérisation de l'amplificateur à fibre (Fig. 23), à savoir : une fibre optique dopée à l'erbium (longueur $=20 \mathrm{~m}$ ), une diode laser de pompe (longueur d'onde $=980 \mathrm{~nm}$ ), une diode laser de signal (longueur d'onde $=1550 \mathrm{~nm}$ ), un isolateur, un multiplexeur, un démultiplexeur, différents atténuateurs et un détecteur à gain variable. Pour les raisons déjà évoquées, cette étude se fait également au moyen de diodes laser émettant une longueur d'onde de $660 \mathrm{~nm}$. 


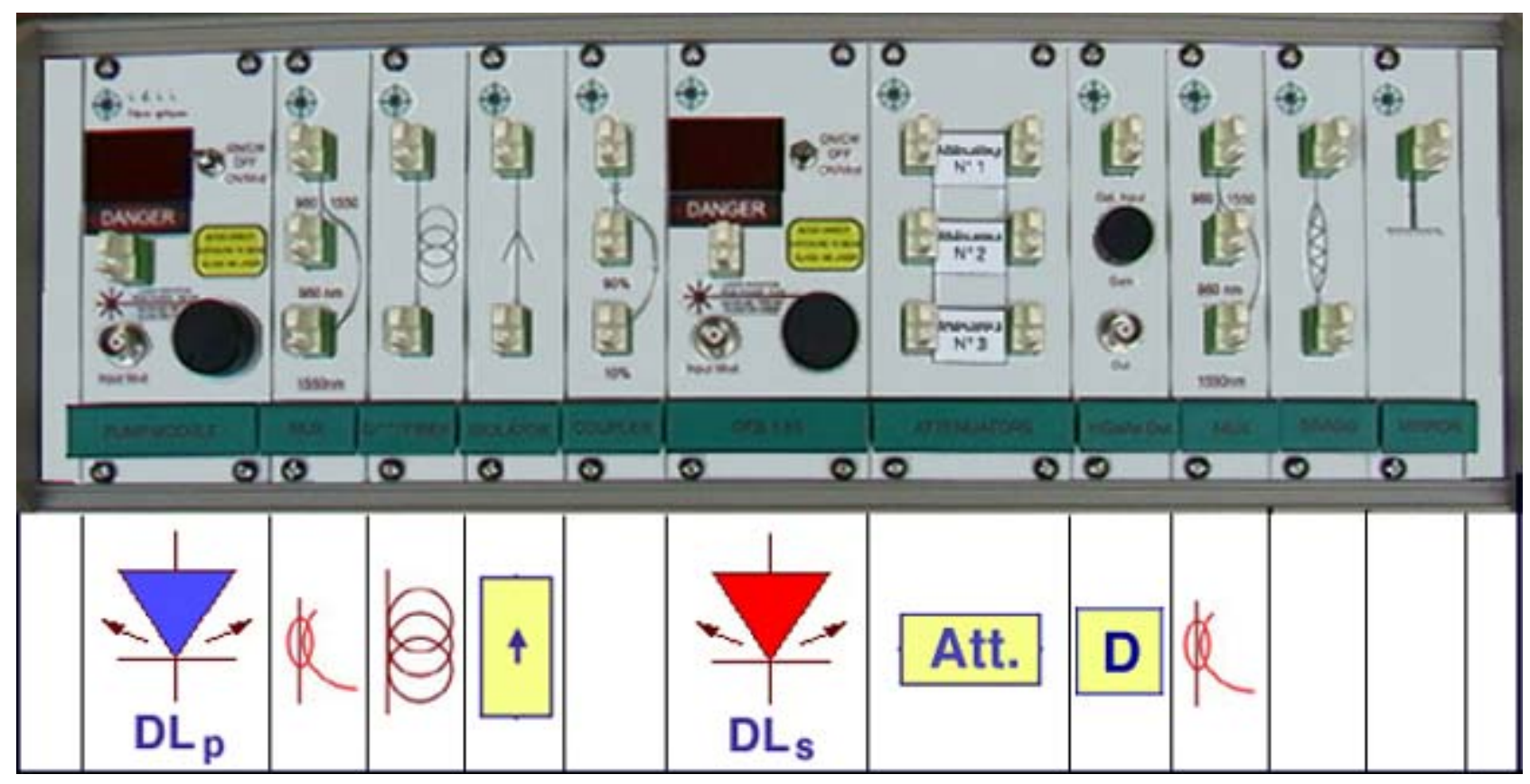

Fig. 23. Amplificateur à fibre optique. Vue de la facade (haut) et schémas correspondant aux différents éléments utilisés dans ce TP (bas). De gauche à droite : diode laser de pompe, multiplexeur, fibre dopée à l'erbium, isolateur, diode laser de signal, atténuateurs, détecteur et démultiplexeur.

L'ajout d'un simple voltmètre en sortie du détecteur permet la détermination de diverses caractéristiques telles que les courants de seuil et les rendements différentiels des deux diodes laser, les conditions de transparence de la fibre dopée ou de l'amplificateur et les coefficients d'atténuation ou de gain des différents éléments ou associations d'éléments (Fig. 24).

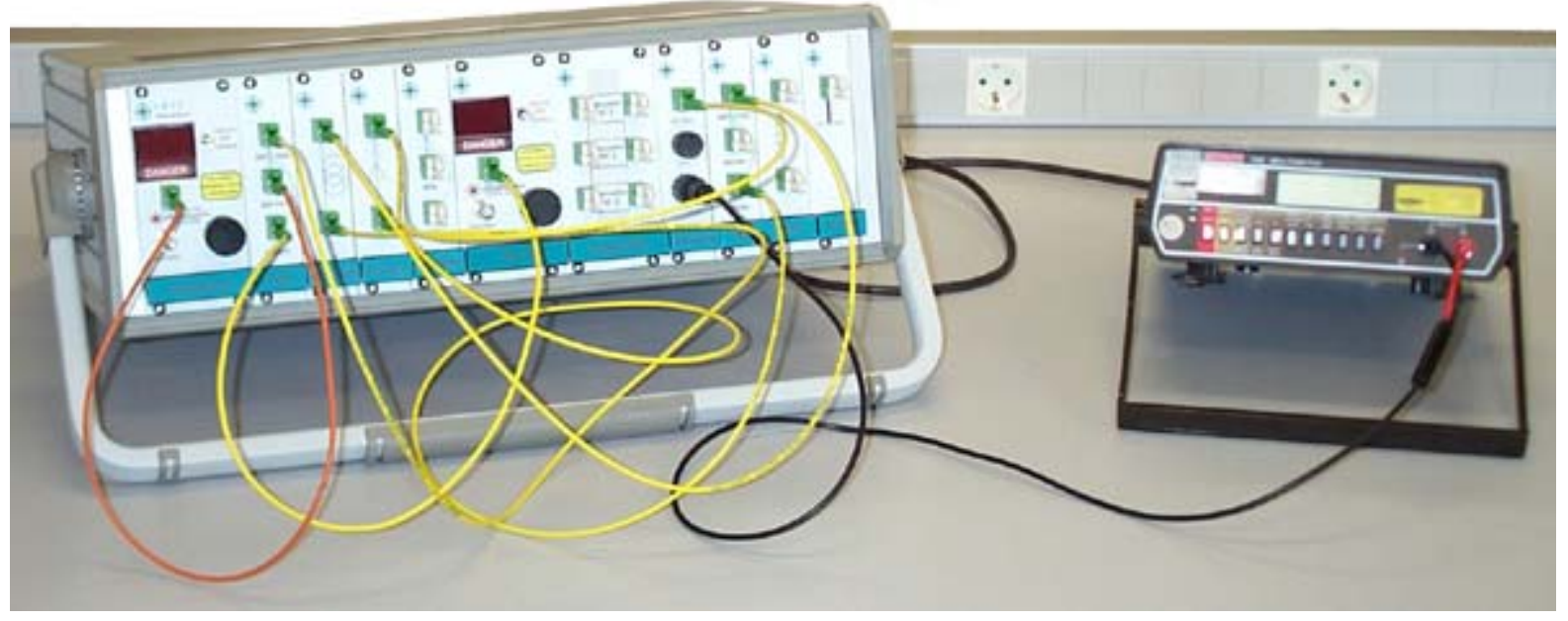

Fig. 24. Vue d'ensemble du montage expérimental.

Nous étudierons successivement :

- les diodes laser de signal et de pompe ;

- les éléments passifs ;

- la fibre dopée Erbium ;

- l'amplificateur dans sa globalité. 


\subsection{Caractérisation des diodes laser}

L'objectif de cette première partie est de déterminer la caractéristique $\mathrm{P}(\mathrm{I})$ des deux diodes laser utilisées dans le montage et d'en déduire la valeur des courants de seuil correspondants. Les valeurs absolues des puissances optiques sont obtenues par la conversion des tensions délivrées par le détecteur à l'aide d'un point d'étalonnage que l'on fournit aux étudiants.
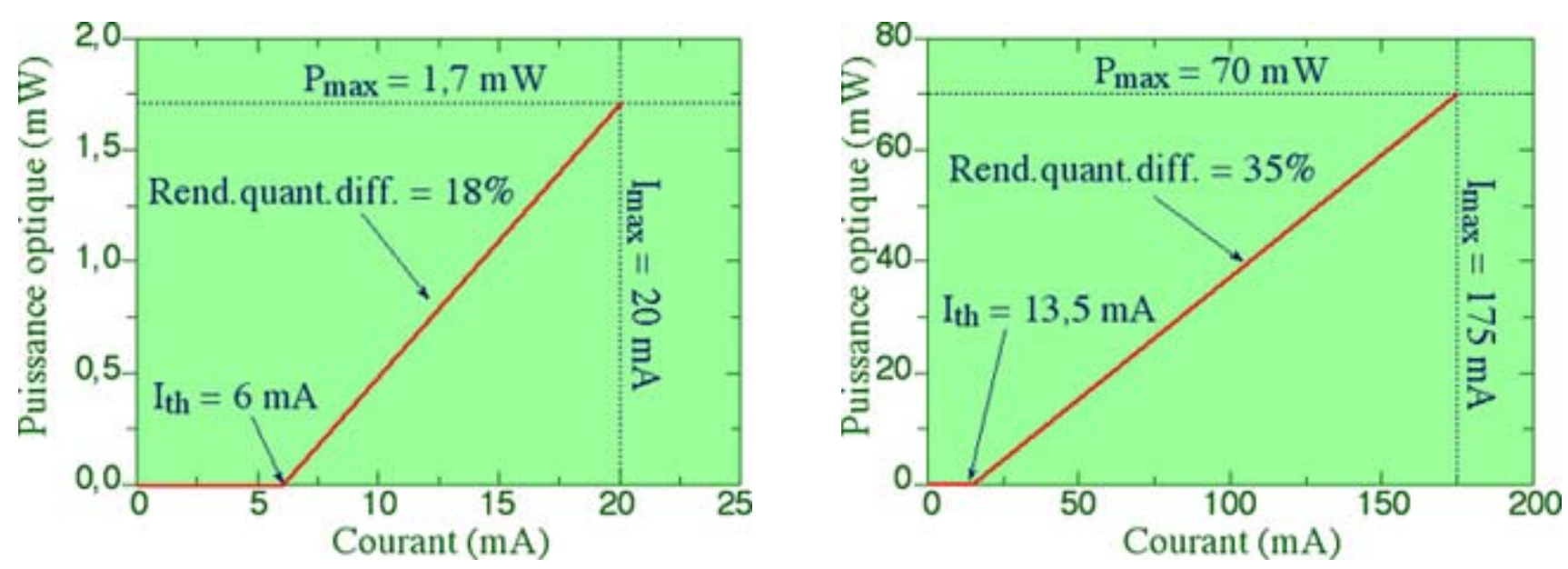

Fig. 25. Caractéristiques P(I) de la diode laser de signal (gauche) et de la diode laser de pompe (droite).

La comparaison des caractéristiques ainsi déterminées (Fig. 25) met en évidence des différences importantes dues à la nature et à la fonction spécifique de chacune des deux sources. Ainsi, lorsqu'on passe de la diode laser de signal à la diode laser de pompe, la valeur de la puissance maximum émise est multipliée par 40 pour un courant multiplié par 9 et le courant de seuil ainsi que le rendement quantique différentiel sont multipliés par un facteur 2 .

\subsection{Caractérisation des éléments passifs}

Dans la démarche visant à « construire » et à caractériser l'amplificateur à fibre, nous proposons d'effectuer, en premier lieu, la caractérisation des éléments passifs indispensables à son fonctionnement. D'après le schéma de

l'amplificateur envisagé (Fig. 26), ces éléments passifs sont au nombre de trois : un isolateur évitant toute réflexion parasite du faisceau de signal vers son propre émetteur, un multiplexeur permettant de coupler le faisceau de la diode laser de pompe dans la fibre dopée à l'erbium et un démultiplexeur pour un contrôle éventuel de la partie du faisceau de pompe qui n'a pas été absorbée durant la traversée de la fibre dopée.

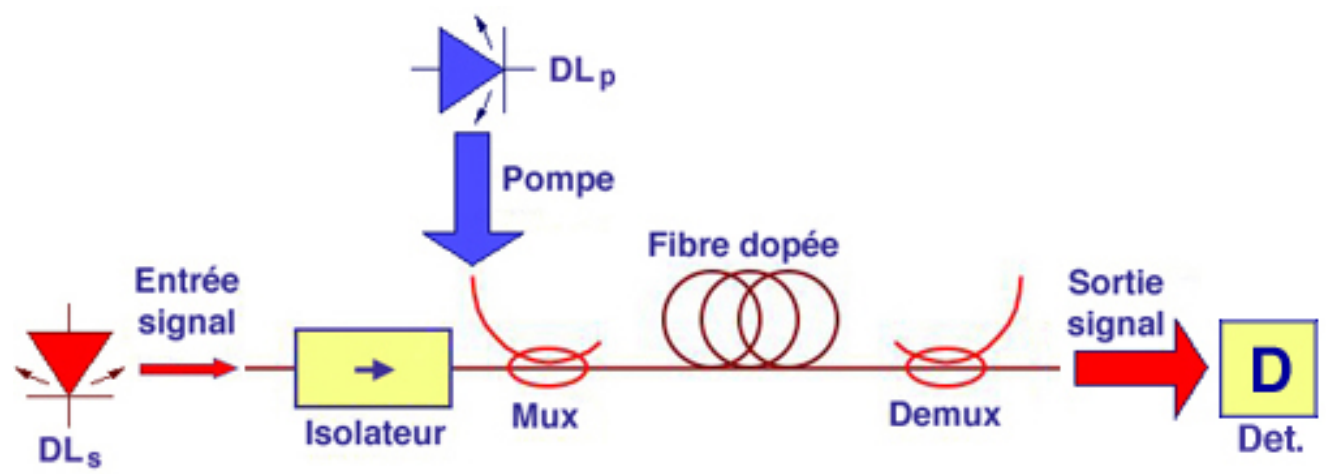

Fig. 26. Schéma de l'amplificateur optique à fibre dopée à l'erbium.

Ici, les trois éléments passifs sont connectés en série (Fig. 27) et on mesure leur coefficient d'atténuation total pour la longueur d'onde du signal optique $(1550 \mathrm{~nm})$. Cette donnée sera exploitée ultérieurement. 


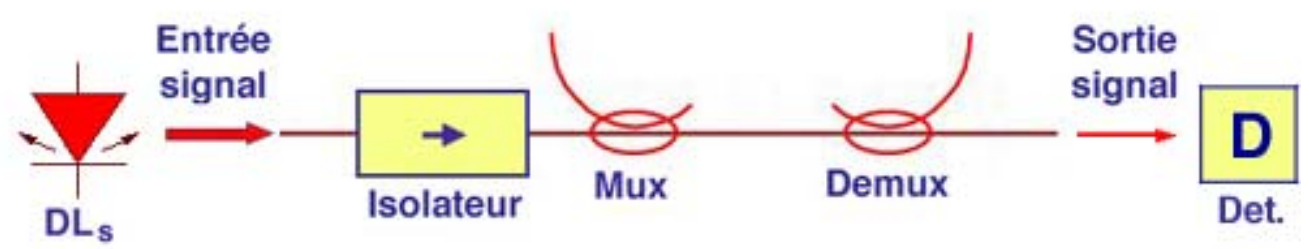

Fig. 27. Montage pour la mesure de l'atténuation des éléments passifs.

\subsection{Caractérisation de la fibre dopée}

La fibre dopée à l'erbium est le cœur de l'amplificateur. Cette fibre montre différents comportements en fonction des rayonnements qui la traversent. On a donc choisi de la caractériser ici dans deux types de fonctionnements dont un correspond à un élément passif et l'autre à un élément actif.

Dans un premier temps, on injecte le faisceau de signal dans la fibre dopée et on mesure la tension délivrée par le détecteur (Fig. 28a). En conservant les conditions d'émission et de détection, on remplace la fibre dopée par une série d'atténuateurs calibrés (Fig. 28b). Par comparaison des deux situations, on déduit le coefficient d'atténuation linéique de la fibre dopée « passive » dont on connaît la longueur.

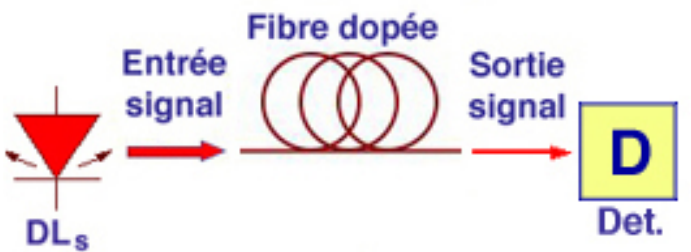

a)

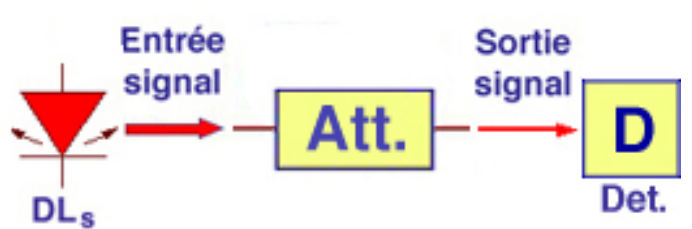

b)

Fig. 28. a) Mesure de la puissance de sortie pour un signal d'entrée donné.

b) Mesure de l'atténuation pour des conditions analogues à celles de la figure 28a.

Dans un deuxième temps, et selon le schéma de l'amplificateur optique, on rajoute les éléments passifs vus précédemment et on connecte la diode laser de pompe au multiplexeur. On recherche alors la valeur de la puissance de pompe qui permet de retrouver, en sortie de l'amplificateur, la puissance du signal obtenue lors de la caractérisation des trois éléments passifs (Fig. 29). Ainsi, on définit les conditions de transparence de la fibre dopée devenue « active ».

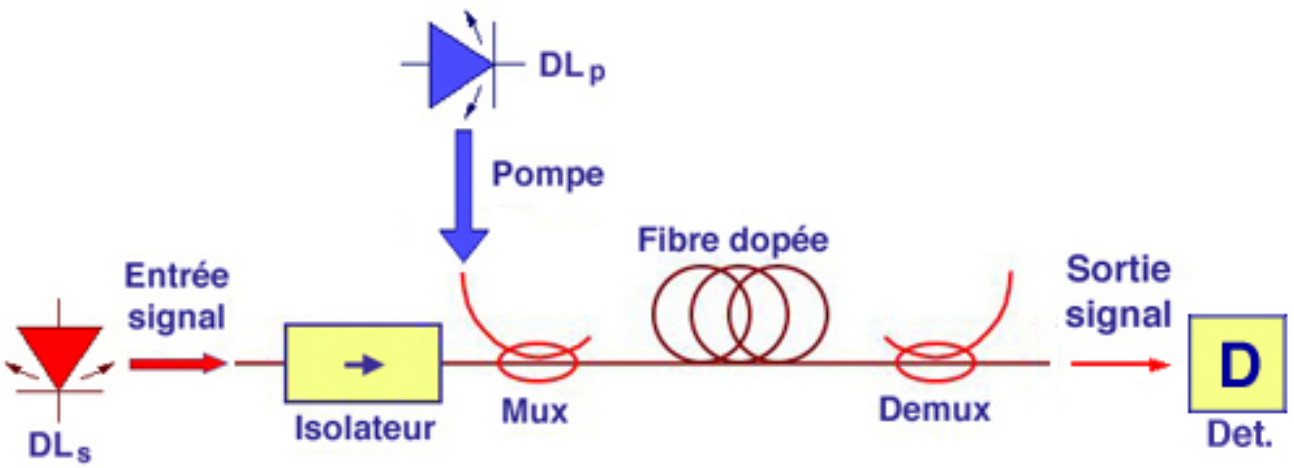

Fig. 29. Dispositif utilisé pour l'étude des conditions de transparence de la fibre dopée.

Il est maintenant naturel d'envisager la caractérisation de l'amplificateur pris dans sa globalité. C'est l'objet de la dernière partie de cette approche expérimentale. 


\subsection{Caractérisation de l'amplificateur à fibre}

Pour permettre aux étudiants de différencier les caractéristiques de la fibre dopée et de l'amplificateur, on précise la valeur de leur gain respectif dans le cas de la transparence de la fibre. On définit ensuite les conditions de transparence de l'amplificateur (Fig. 30) et on détermine les nouvelles valeurs des gains correspondants.

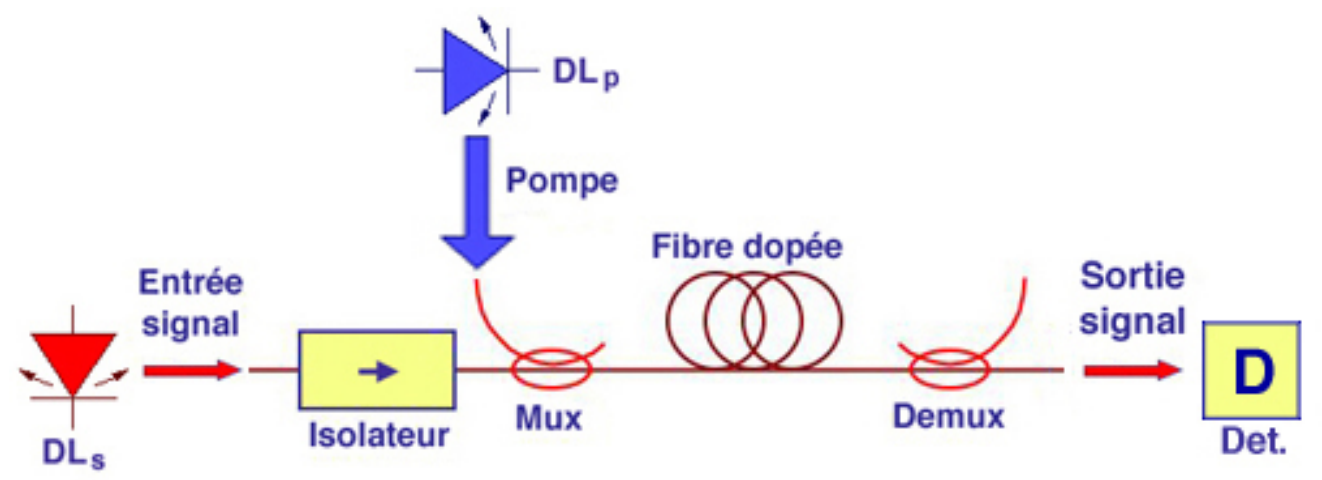

Fig. 30. Dispositif utilisé pour l'étude des conditions de transparence de l'amplificateur.

En utilisant les atténuateurs calibrés, on détermine les paramètres de pompage permettant d'obtenir des valeurs de gain données (Fig. 31). Enfin, on détermine la valeur maximum du gain de l'amplificateur dans la configuration étudiée.

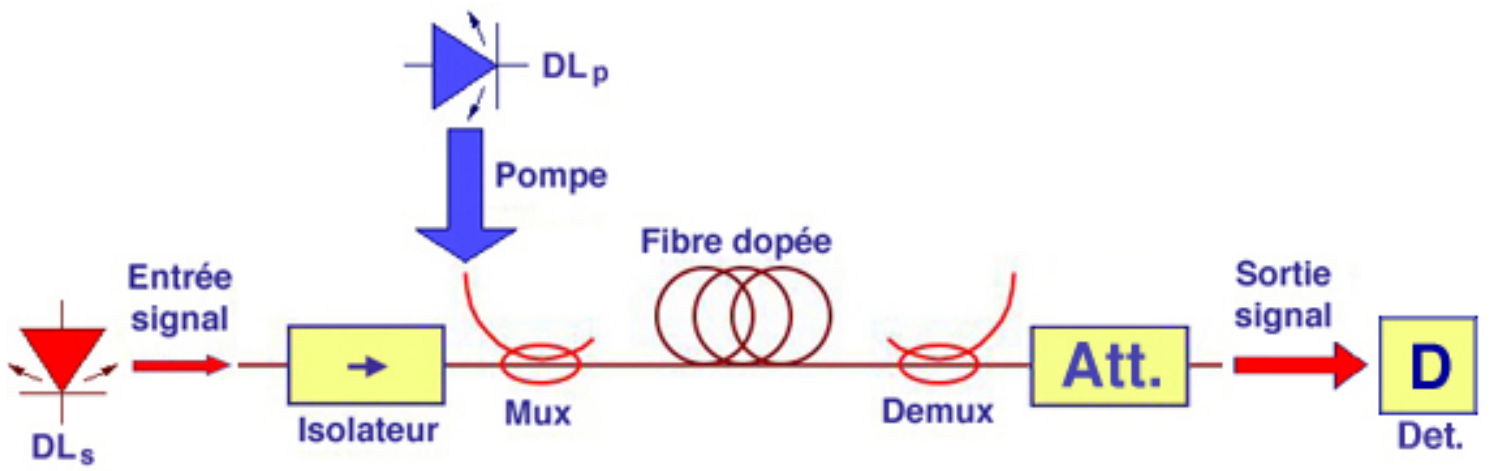

Fig. 31. Dispositif permettant l'étude du fonctionnement de l'amplificateur à différentes valeurs de gain.

\section{Conclusion}

Grâce aux nombreuses notions abordées et à la variété des mesures réalisées, les trois séries de manipulations que l'on vient de présenter permettent d'appréhender concrètement diverses problématiques liées au domaine des télécommunications optiques.

Dans une démarche à la fois pédagogique, de sécurité et d'économie, nous avons montré qu'un appareillage courant et bon marché permettait d'étudier les principales caractéristiques d'éléments fondamentaux d'une liaison par fibre optique tels que l'émetteur (diode laser), la fibre optique et l'amplificateur à fibre dopée.

\section{Références bibliographiques}

[1] G. Hée et al., Les lasers. Risques et préventions, Cahiers de notes documentaires - Hygiène et sécurité du travail, 173 (1998). 


\section{Annexe. Les classes de risques des lasers [1]}

De façon générale, les lasers sont répartis dans différentes classes de risques en fonction du danger potentiel de leur rayonnement accessible :

- Classe 1 : lasers intrinsèquement inoffensifs dans toutes les conditions d'utilisation.

- Classe 2 : lasers n'émettant que dans le spectre visible avec une puissance maximale de $1 \mathrm{~mW}$. Une observation directe du faisceau, même avec des moyens optiques, n'entraîne pas de lésion oculaire. Toutefois, le réflexe palpébral (réflexe de fermeture de la paupière) ne doit pas être entravé.

- Classe 3A : pour les lasers émettant dans le spectre visible, la protection de l'œil est assurée par le réflexe palpébral, et pour les autres longueurs d'onde, le risque pour l'œil ne doit pas être supérieur à celui de la classe 1. En lumière visible, la puissance maximale est de $5 \mathrm{~mW}$ et l'éclairement énergétique en un point quelconque du faisceau doit être inférieur à $25 \mathrm{~W} \cdot \mathrm{m}^{-2}$. L'observation directe du faisceau à l'aide de moyens optiques peut être dangereuse.

- Classe 3B : lasers dont la puissance maximale est de $500 \mathrm{~mW}$. L'observation directe du faisceau est toujours dangereuse. L'observation de réflexions diffuses est normalement sans danger pour des temps d'exposition inférieurs à $10 \mathrm{~s}$.

- Classe 4 : lasers ne répondant pas aux conditions des classes 1, 2, 3A ou 3B. Il n'y a pas de limite supérieure de puissance pour les lasers de cette classe. Leur rayonnement et les réflexions, même diffuses, présentent un danger important pour les yeux et la peau.

Afin d'illustrer cette classification des risques, nous présentons quelques exemples de sources laser émettant dans la gamme des $660 \mathrm{~nm}$ et voisines des sources utilisées en TP (Fig. 32). Toutefois, contrairement aux diodes laser dont nous proposons l'étude, on notera que les sources présentées ici produisent un faisceau collimaté qui, de ce fait, est potentiellement plus dangereux.
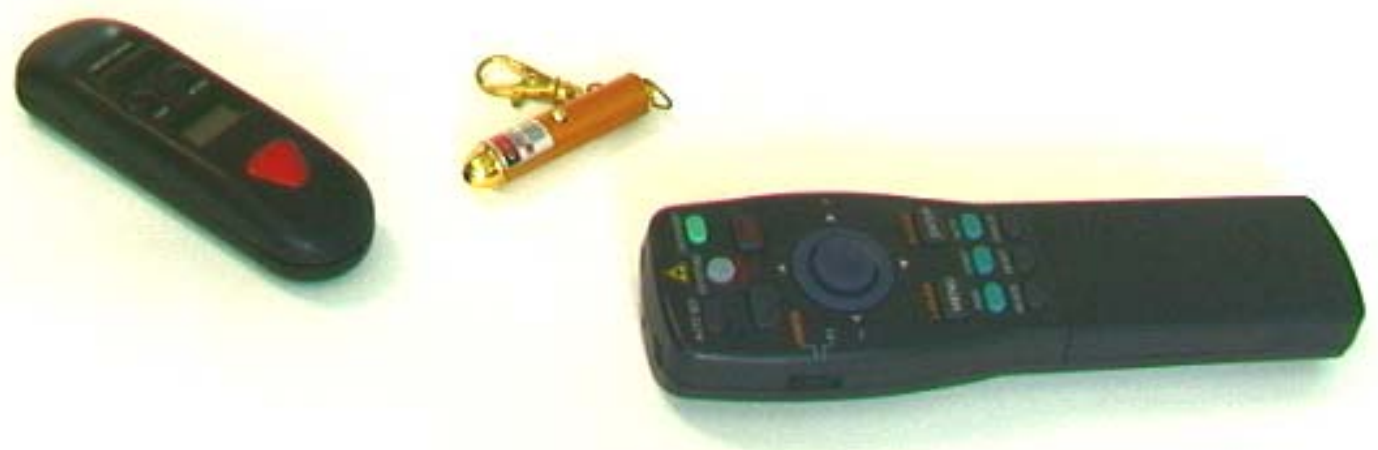

Fig. 32. Exemples de sources lasers. Gauche : $\mathrm{P}_{\max }=5 \mathrm{~mW}$, classe $3 \mathrm{~A}$; centre : $\mathrm{P}_{\max }=1 \mathrm{~mW}$, classe 2 ; droite $: \mathrm{P}_{\max }=1 \mathrm{~mW}$, classe 2 .

\section{Calcul de l'éclairement énergétique}

Dans le cas d'un laser émettant un rayonnement visible, l'éclairement énergétique $\mathrm{E}_{\mathrm{c}}$ au niveau de l'œil peut se calculer à partir des caractéristiques du laser et de la distance d'observation, au moyen de l'expression suivante :

$$
\mathrm{E}_{\mathrm{c}}=1,27 \mathrm{P} /(\mathrm{d}+\mathrm{aR})^{2}
$$

où $\mathrm{P}$ est la puissance du laser, $\mathrm{d}$ est le diamètre du faisceau à la source, a est l'angle de divergence du faisceau en radian, et $\mathrm{R}$ est la distance d'observation.

Pour les diodes laser étudiées en TP ( $\left.\mathrm{P}_{\max }=5 \mathrm{~mW}, \mathrm{~d} \sim 3 \mu \mathrm{m}, \mathrm{a} \sim 0,35 \mathrm{rd}\right)$ et pour une distance d'observation $\mathrm{R}=20 \mathrm{~cm}$, le calcul de l'éclairement énergétique pour une observation directe du faisceau conduit à une valeur voisine de $1,3 \mathrm{Wm}^{-2}$, ce qui est largement inférieur à la limitation de la classe $3 \mathrm{~A}$. L'observation de réflexions diffuses ne présente alors aucun danger. 